\title{
Effect of cytokine-induced killer cells combined with dendritic cells on the survival rate and expression of 14-3-3 $\zeta$ and p-Bad proteins in Lewis lung cancer cell lines
}

\author{
YANG HOU ${ }^{1}$, DONGYU ZANG ${ }^{2}$, XIAOMING LI $^{3}$ and FUZHI LI ${ }^{2}$ \\ ${ }^{1}$ Life Science Institute of Jinzhou Medical University; ${ }^{2}$ Department of Thoraxes Surgery of The Third Affiliated Hospital, \\ Jinzhou Medical University; ${ }^{3}$ Department of Histology and Embryology, Jinzhou Medical University, \\ Jinzhou, Liaoning 121001, P.R. China
}

Received October 29, 2016; Accepted February 23, 2018

DOI: $10.3892 / \mathrm{ol} .2018 .8834$

\begin{abstract}
In the present study, the function and mechanism of cytokine-induced killer cells (CIK) combined with dendritic cells (DC-CIK) were examined in Lewis lung cancer (LLC) cells. Co-culture of CIK dendritic cells (DC) in vitro was used to investigate their proliferation and the antitumor effects on LLC cells. DC and CIK cells were collected from healthy human peripheral blood mononuclear cells and co-cultured as an experimental group, while LLC cells were cultured alone as a control group. Cell morphology was observed by an inverted microscope and an MTT assay was utilized to detect the proliferation of LLC cells. Expression of 14-3-3 $\zeta$ and $\mathrm{p}$-Bad were measured by western blot analysis. Compared with the control group, treatment of LLC cells with DC-CIK resulted in decreased cell adherence, reduced cell proliferation and abnormal morphological changes. Additionally, DC-CIK treatment of LLC cells resulted in the decreased expression of 14-3-3 $\zeta$ and p-Bad protein in LLC cells, which may provide important information pertaining to the possible mechanism of DC-CIK-induced antitumor activity against LLC cells. The present study provides a theoretical and experimental basis for the clinical treatment of DC-CIK cell co-culture.
\end{abstract}

\section{Introduction}

An estimated 1.8 million novel lung cancer cases occurred in 2012 , accounting for $\sim 13 \%$ of total cancer diagnoses worldwide (1). Sundar et al (2) demonstrated that there are two main types of lung cancer, small-cell lung cancer (SCLC) and non-small-cell lung cancer (NSCLC), with $>80 \%$ of lung cancer

Correspondence to: Dr Fuzhi Li, Department of Thoraxes Surgery of The Third Affiliated Hospital, Jinzhou Medical University, 40 Section 3, Songpo Road, Jinzhou, Liaoning 121001, P.R. China E-mail: 124011334@qq.com

Key words: cytokine-induced killer cells, dendritic cells, Lewis lung cancer cell, phosphorylated-Bad cases being NSCLC in 2014. The combination of the traditional methods of surgery, chemotherapy and radiotherapy with immunotherapy is a novel modality for anti-cancer therapies to reduce the mortality of patients with cancer. However, there are still unacceptably high rates of relapse and mortality in patients with early-stage, surgically-resectable lung cancer (3). Currently, chemotherapy is the standard treatment for advanced stage and metastatic NSCLC (4). However, chemotherapy is associated with a decline in sensitivity over time and often has a toxicity profile that reduces the overall quality of life of the patients, without significantly improving prognosis (5). Despite numerous advances in treatment modalities, the treatment and mechanism for NSCLC progression remains unclear.

Dendritic cells (DCs) are the most effective antigenpresenting cells and have been applied in cellular immunotherapy research worldwide (6). Since the first DC vaccine for prostate cancer was approved by the FDA, DC-based immunotherapy has become an increasingly promising novel therapeutic option. Cytokine-induced killer cells (CIK) are well known for their antitumor activity (7). In recent years, there has been an upsurge of interest in unraveling the roles of combined DC-CIK therapy on NSCLC, with numerous results indicating that $\mathrm{DC} / \mathrm{CIK}$ immunotherapy combined with other treatments has a good clinical efficacy and prospects for the treatment of NSCLC (8-11). However, the mechanism by which DC-CIK cells can specifically kill NSCLC remains unclear. Therefore, the present study used DCs to induce CIK cells specifically targeted to NSCLC.

The family of 14-3-3 proteins serve key roles in integrating cellular survival signaling. $14-3-3 \zeta$ is a member of a family highly conserved proteins that control key aspects of cellular function, including proliferation, apoptosis, and cell survival (12). Experimental and clinical results from previous studies have suggested that 14-3-3 proteins represent an addiction for numerous cancers and consequently are an attractive target for anti-cancer therapeutics $(13,14)$. The protein has been identified as a putative oncoprotein in several cancers, including NSCLC, liver cancer, head and neck squamous cell carcinoma, and is a potential target for developing a prognostic biomarker and therapeutics that may enhance the antitumor activity of cisplatin for the treatment of NSCLC (15). 
An increasing amount of evidence has indicated that dysregulation of apoptosis contributes to the development of human cancers (16). Bad, a proapoptotic Bcl-2 family protein regulates the intrinsic apoptosis pathway, Bad is also regulated by phosphorylation, which leads to its sequestration by 14-3-3 scaffold proteins (17). Phosphorylated (p)-Bad dissociates from Bcl-2 and is sequestered in the cytosol to promote cell survival (18).

In the present study the antitumor effects of DC-CIK cells in Lewis lung cancer (LLC) cell lines were evaluated and the underlying mechanism of these effects was investigated. The DC-CIK cells effects on cytotoxic potentiation and apoptosis were investigated and the cytotoxic effects were evaluated using an MTT assay and apoptosis morphology observation. Expression of 14-3-3 $\zeta$ and p-Bad were measured by western blot analysis.

\section{Materials and methods}

Culture of CIK. Peripheral blood mononuclear cells (PBMCs) were collected from healthy blood donors ( 2 males, 2 females; median age, 39 years; age range, 28-50 years) with no clinical symptoms of any disease. A total of $10 \mathrm{ml}$ of blood was collected from each donor in evacuated tubes containing $0.1 \mathrm{mg} / \mathrm{ml}$ heparin. Mononuclear cells (MNC) were isolated with lymphocyte separating medium (Wuhan Huamei Biotech Co., Ltd., Wuhan, China) and washed by normal saline (centrifugation, 1,341.48 x g for $15 \mathrm{~min}$ ). Mononuclear cells were cultured in Iscove's modified Dulbecco's medium (IMDM) supplemented with 10\% fetal calf serum (Hyclone; GE Healthcare Life Sciences, Logan, UT, USA) and recombinant human (rh) interferon (IFN) $-\gamma$ (Santa Cruz Biotechnology, Inc., Dallas, TX, USA) 1,000 U/ml, CD3 monoclonal antibody (sc-20047; 1:4,000, final concentration, 50 ng/ml; Santa Cruz Biotechnology, Inc.) and 300 units/ml of recombinant human interleukin-2 (rh IL-2) were added to CIK cells and cultured at $37^{\circ} \mathrm{C}$ in $5 \% \mathrm{CO}_{2}$ incubator.

Medium was changed every three days for half dose and added with $300 \mathrm{U} / \mathrm{ml}$ rh IL-2 to maintain the cell concentration at $1 \times 10^{6} / \mathrm{ml}$. On day 14 , CIK cells were harvested with trypsin and washed twice with PBS. Ethical approval for the present study was obtained from the Ethics and Welfare Committee of Jinzhou Medical University (Jinzhou, China). Written informed consent was obtained from all participants.

Culture of DC. PBMC were cultured in serum-free RPMI-1640 medium (Hyclone; GE Healthcare Life Sciences) at $37^{\circ} \mathrm{C}$ in $5 \% \mathrm{CO}_{2}$ for $2 \mathrm{~h}$ and washed by serum-free RPMI 1640 culture medium twice. Adherent cells were suspended in RPMI 1640 culture medium which contained rh Granulocyte-macrophage colony-stimulating factor (GM-CSF) $(1,000 \mathrm{U} / \mathrm{ml})$, rhIL-4 $(500 \mathrm{U} / \mathrm{ml})$, rh tumor necrosis factor (TNF)- $\alpha(200 \mathrm{U} / \mathrm{ml})$ for 7 days.

DC co-cultured with CIK. Mature DCs were obtained from the aforementioned culture of DC 7 days after GM-CSF TNF- $\alpha$ induction, then CIK and DCs were co-cultured in RPMI-1640 at $37^{\circ} \mathrm{C}$ in $5 \% \mathrm{CO}_{2}$ in a proportion of 1:5. Cells were cultured together for 3 days.
Culture of LLC cells. LLC cells were obtained from Shanghai Institute of Biochemistry and Cell Biology (Chinese Academy of Sciences, Shanghai, China). Cells were cultured in RPMI-1640 medium supplemented with $10 \%$ heat inactivated fetal bovine serum (Hyclone; GE Healthcare Life Sciences) and antibiotic solution (penicillin $100 \mathrm{U} / \mathrm{ml}$ and streptomycin $100 \mu \mathrm{g} / \mathrm{ml}$ ). (Sigma-Aldrich; Merck KGaA; Darmstadt, Germany). Cells were cultured under standard conditions in a $5 \% \mathrm{CO}_{2}$ humidified incubator at $37^{\circ} \mathrm{C}$. LLC cells alone were cultured as control group.

Cell migration assay. The cells were divided into 4 groups: DC-CIK, CIK, PBS and the control group (RPMI-1640 medium with $10 \%$ fetal calf serum). RPMI-1640 medium with $10 \%$ fetal calf serum was added in the upper transwell insert chamber with the control group. DC-CIK, CIK cells or PBS were seeded at the concentration of $0.6 \times 10^{5}$ cells/well in the upper transwell insert chamber containing a polycarbonate filter (6.5 mm diameter, $0.4 \mu \mathrm{m}$ pores; Corning Costar, Corning, NY, USA). LLC cells was added to the lower chamber at $2.0 \times 10^{5}$ cells $/ 500 \mu \mathrm{l} /$ well in all groups, and the plates were incubated for 7 days at $37^{\circ} \mathrm{C}$ in $5 \% \mathrm{CO}_{2}$. Both upper and lower chamber cells were cultured in RPMI-1640 medium with $10 \%$ fetal calf serum. Images of the LLC cells in each group were captured under an inverted microscope (Olympus Corporation, Tokyo, Japan) to evaluate the number and morphology of cells for $20 \mathrm{~min}$ at room temperature at 3,5 and 7 days (magnification, $\mathrm{x} 200$ ). LLC cells were used as target cells, and the CIK and DC-CIK cells cultured for 7 days were used as effector cell mixed for the cell viability assay and western blot analysis.

Cell viability assay. The cell viability of LLC cells was measured in the 7th day. LLC cells were obtained from the aforementioned cell migration assay were diluted with RPMI-1640 medium containing 10\% fetal calf serum at $1 \times 10^{4}$ cells $/ \mathrm{ml}$. There were three parallel wells for DC-CIK, CIK, PBS and the control group. The MTT assay was employed to examine cell viability. Briefly, MTT was added to the culture medium at a final concentration of $0.5 \mathrm{mg} / \mathrm{ml}$ and cells were incubated at $37^{\circ} \mathrm{C}$ for $3 \mathrm{~h}$, the culture medium containing MTT was removed. Dimethyl sulfoxide (100 $\mu \mathrm{l})$ was then added into each well to dissolve the formed blue formazan. Absorbance (A) was read at $490 \mathrm{~nm}$ on a microplate reader. Cell viability was calculated as follows: Cell viability $(\%)=\mathrm{A}$ experiment group/A control group x100\%.

Western blot analysis. LLC cells were lysed with RIPA lysis buffer [50 mM of tris- $\mathrm{HCl}(\mathrm{pH} 7.5), 150 \mathrm{mM}$ of $\mathrm{NaCl}$, $1 \%$ NP-40, $1 \%$ sodium deoxycholate, $0.1 \%$ SDS, $5 \mathrm{mM}$ of EDTA, $25 \mathrm{mM}$ of NaF, $2 \mathrm{mM} \mathrm{Na} 3 \mathrm{VO} 4$, and $1 \mathrm{mM}$ of PMST] containing 1:100 diluted protease inhibitor cocktail (cat. no. P8340; Sigma-Aldrich; Merck KGaA) on ice. The protein concentrations were measured using a Bradford assay subsequent to centrifugation at $13,000 \mathrm{x}$ g for $15 \mathrm{~min}$ at $4^{\circ} \mathrm{C}$. Equal amounts of proteins were separated on a $10 \%$ gel by SDS-PAGE and transferred to a polyvinylidene fluoride membrane (Bio-Rad Laboratories, Inc., Hercules, CA, USA). For western blot analysis of 14-3-3६, (ab87361; 1:1,000; Abcam, Cambridge, MA, USA), p-bad (ab171725; 1:1,000; Abcam) and $\beta$-actin (A5441; 1:10,000; Sigma-Aldrich; Merck 
$\mathrm{KGaA}$ ), the primary antibodies used in the experiment were probed and incubated overnight at $4^{\circ} \mathrm{C}$, followed by secondary antibody reactions with horseradish peroxidase-cinjugated goat anti-mouse IgG (ab205719; 1:5,000; Abcam) for $1.5 \mathrm{~h}$. The detection was evaluated by the 3-bromo-4-chloro-5-indolyl phosphate and nitro blue tetrazolium reaction (Ameresco, Inc., Framingham, MA, USA).

Statistical analysis. All statistical analyses were performed using SPSS Statistics 17.0 (SPSS, Inc., Chicago, IL, USA). Data are expressed as the mean \pm standard deviation. One-way analysis of variance was used followed by Fisher's Least Significant Difference test for homogeneous data and followed by Dunnett's T3 for the heteroscedastic data. $\mathrm{P}<0.05$ was considered to indicate a statistically significant difference.

\section{Results}

LLC proliferation. Adherent LLC cells that exhibited a round or semi-shuttle shape growth were examined under an inverted microscope (magnification, x200) at 24-48 h after they were passaged (Fig. 1A). Proliferation began at the first three days following culturing. The cells of passaged 2 exhibited a shuttle or fibroblast shape, with the cells being fully extended (magnification, x400; Fig. 1B).

Morphology of DCs and CIK cells. Microscopic observation revealed that adherent DCs were visible at the bottom of the plate. On day 7, the cells exhibited an irregular, fusiform or stellate shape that is characteristic of mature DCs. The DCs were thriving, with evident thick and long dendritic protrusions (Fig. 2A). CIK cells were spherical, uniform and transparent, cells were increased in size and small colonies had formed. On day 12, the number of colonies had markedly increased, exhibiting cellular proliferation (Fig. 2B).

Morphology of LLC cells following co-culture with DC-CIK. LLC cellular morphology did not change significantly in all groups at 3 day of co-culture. Transparent LLC cells with a uniform size in the CIK group were superior to the DC-CIK group. LLC cells exhibited inflation, cytoplasmic contraction and an increase in intercellular space in the DC-CIK group at 5 day of cell co-culture (Fig. 3A). LLC cells shrank in size and the intercellular space continued to increase in the CIK cells group at 7 day (data not shown). A proportion of LLC cells exhibited cell death in the DC-CIK group. Cell morphology change was evident, and cell size became smaller and further away from the surrounding cells. Connections between cells also disappeared (Fig. 3B). These images demonstrate that DC-CIKs induced morphological changes in LLC cells.

$D C$-CIK inhibited LLC cell viability. In order to examine the cytotoxic effects of DC-CIK on LLC cells, cell viability was examined by an MTT assay after LLC cells were incubated with PBS, CIK or DC-CIK for 7 days. The DC-CIK group was statistically different from the other groups. The results demonstrated that DC-CIK inhibited the proliferation of LLC cells $(\mathrm{P}<0.01$; Fig. 4).
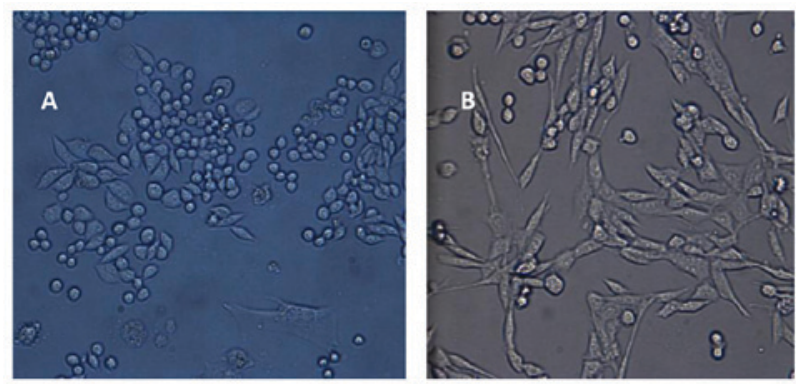

Figure 1. Photographs of morphology. LLC cells were passaged every other day. (A) LLC cells of 24-48 $\mathrm{h}$ after they were passaged (magnification, $\mathrm{x} 200$ ). LLC cells were round or semi-shuttle shape. (B) LLC cells past passage 2 became shuttle or fibroblast shape (magnification, x400). LCC, Lewis lung cancer.
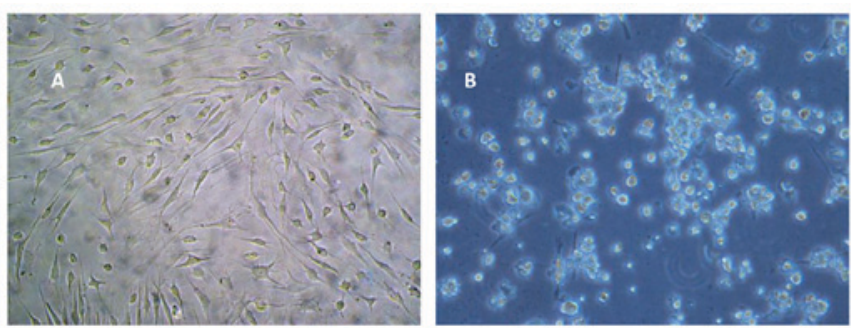

Figure 2. Morphology of DC and CIK cells on the 7th and 12th (B) day of proliferation, respectively (magnification, x400). (A) On day 7 of culture DCs were thriving, with evident thick and long dendritic protrusions. (B) CIK cells exhibited good refraction, suspended growth. Small colonies had formed. On day 12 , the number of colonies had markedly increased demonstrating that the cells had proliferated. DC, dendritic cell; CIK, cytokine-induced killer.
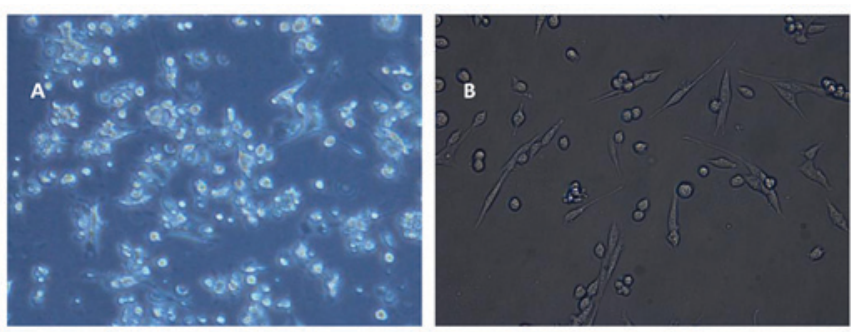

Figure 3. Morphology of LLC cells on 5th (A) and 7th (B) day in the DC-CIK group, respectively. These images show that DC-CIK induced morphological changes in LLC cells (magnification, x200).

Effect of DC-CIK on expression levels of 14-3-3६ and p-Bad. To illustrate the mechanism of DC-CIK induced apoptosis, the involvement of 14-3-3 $\xi$ and p-Bad were investigated by western blot analysis. As depicted in Fig. 5, compared with the control group, the protein level of 14-3-3 $\zeta$ and p-Bad was slightly decreased in the DC-CIK group. The results demonstrate that DC-CIK reduced the expression of $14-3-3 \xi$ and p-Bad protein in LLC cells.

\section{Discussion}

NSCLC is the leading cause of cancer-associated mortality in the USA (15). To date, surgery, radiotherapy and chemotherapy are still the principal therapeutic regimens; however, radiotherapy and chemotherapy exhibit heavy toxic side effects, which are detrimental to the survival rate of patients (19). 


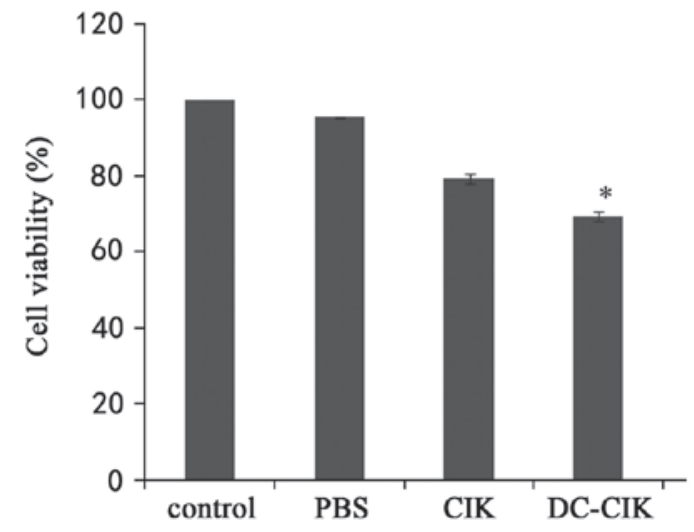

Figure 4. The effect of DC-CIK on the viability of LLC cells. LLC cells were treated with CIK, DC-CIK for 7 days. Cell viability was measured using the MTT assay. DC-CIK inhibited the proliferation of LLC cells. The results are expressed as the mean \pm standard deviation. ${ }^{*} \mathrm{P}<0.01$ vs. control, PBS CIK. DC, dendritic cell; CIK, cytokine-induced killer cell; LLC, Lewis lung cancer.
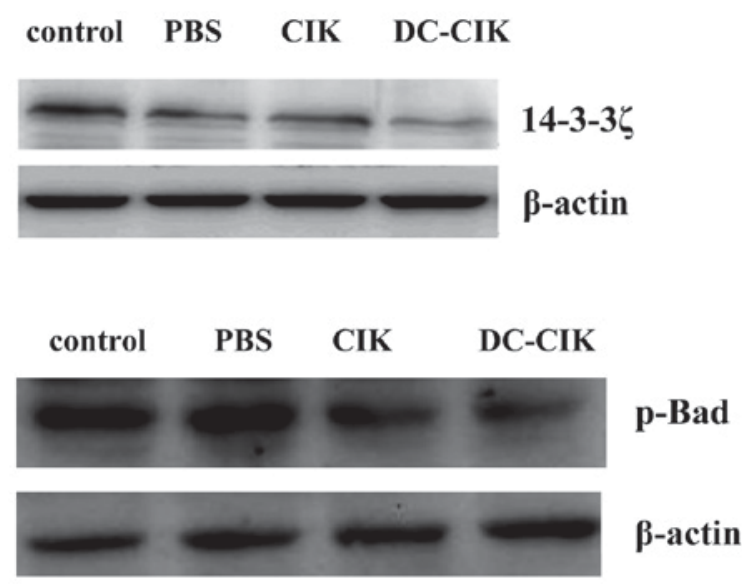

Figure 5. Western blotting data demonstrating the changes of $14-3-3 \xi$ and p-Bad expression in LLC cells. DC-CIK therapy downregulated $14-3-3 \zeta$ and p-Bad in LLC cells. $\beta$-actin was used as the internal control. LCC, Lewis lung cancer; DC, dendritic cells; CIK, cytokine-induced killer cell.

The study of Zhou et al (20) demonstrated that a DC vaccine combined with CIK cells induced a T-cell-mediated immune response that included activating native $\mathrm{T}$ cells, which serve a critical role in the innate and adaptive immune responses. In 2005, Lee et al (21) confirmed the curative effect of a DC vaccine pulsed with autologous tumor lysate in patients with hepatocellular carcinoma. These findings demonstrate that DC/CIK cells can be effective against a variety of cancers through immunotherapy. A previous study was conducted with the goal of improving the efficacy of DC-based immunotherapy (7). The combined DC-CIK therapy, with synchronous radiotherapy and chemotherapy to treat stage IIIB non-small cell lung cancer was superior to single synchronous radiotherapy and chemotherapy (22). DC-CIK combined with concurrent radiochemotherapy was demonstrated to improve the life quality and prolong the survival time of patients (22). In the present study, DC-CIK treatment clearly reduced cell viability on LLC cells by a cell viability assay-this was consistent with the study of Cui et al (23). The present study identified that co-cultured DCs and CIK cells inhibited the proliferation
LLC cells by downregulating 14-3-3 $\zeta$ and p-Bad. DC/CIK cell therapy may be an effective treatment strategy. Similar results have been published by a previous study regarding liver cancer cells (24).

14-3-3 proteins bind to a number of functionally diverse signaling proteins including protein kinases and protein phosphatases, and are involved in important cellular processes such as signal transduction, cell cycle control, and apoptosis $(15,25)$. Ectopic expression of 14-3-3 has been discovered in various malignancies, including lung cancer, liver cancer and head and neck squamous cell carcinoma. Enhanced expression of 14-3-3 proteins have been detected in human cancers including lung cancer (26), which correlates with more aggressive tumors and a poor prognosis (17). Downregulation of $14-3-3 \xi$ in head and neck cancer cells and lung cancer cells renders cells more sensitive to chemotherapy $(27,28)$. The over-expression $14-3-3 \zeta$ in NSCLC tissues is associated with the severity of disease and similarly targeted knock-down of 14-3-3६ using RNAi in A549 cells, and also increased the sensitivity of cells to cisplatin (29). These findings suggest that $14-3-3 \zeta$ serves an important role in promoting tumor aggressiveness. As 14-3-3 $\zeta$ expression is closely associated with NSCLC disease, the present study examined DC-CIK for anti-cancer effects on LLC cells. It was determined that DC-CIK reduced the expression of $14-3-3 \xi$ protein. It is supported by the study of Lin et al (30).

The mitochondria-dependent (type II) apoptotic pathway begins with the apoptosis-regulating protein $\mathrm{Bcl}-2$ family, including the anti-apoptotic proteins Bcl-2 and Bcl-xL in addition to the pro-apoptotic proteins Bad and Bax (31). Previous studies have demonstrated that the effect of apoptosis and repressed cell viability may be due to the decreased levels of p-Bad (29,32). Previous report indicated that the expression of $\mathrm{p}$-Bad was increased in colorectal cancer cells, and suggested that the increased expression of the protein in malignant colorectal epithelial cells compared with the normal mucosal epithelial cells may possibly alter the regulation of cell death during colorectal tumorigenesis (33). However the association between p-Bad and cancers remains controversial. A previous study reported this beauvericin-induced apoptosis in human NSCLC A549 cells was also accompanied by the upregulation of p-Bad (30). $\mathrm{P}-\mathrm{Bad}$ expression was detected well in normal gastric mucosal epithelial cells, whereas it was detected in only $51 \%$ (31 of the 60) of the cancers. The decreased expression of p-Bad in malignant gastric epithelial cells compared with normal mucosal epithelial cells suggested that loss of $\mathrm{p}-\mathrm{Bad}$ expression may serve a role in gastric tumorigenesis (34). In the present study, the results demonstrated that LLC cells treated with DC-CIK resulted in decreased cell adherence and abnormal morphological changes which are characteristics of apoptosis and reduced cell proliferation. In addition, DC-CIK treatment resulted in decreased expression of $\mathrm{p}-\mathrm{Bad}$ protein in LLC cells. Depending on the nature of its target proteins, 14-3-3 binding impacts multiple signaling pathways that determine cell fate and organ development. For example, 14-3-3 associations control Raf signaling fidelity and neutralizes Bad-mediated apoptosis (35). Therefore, further study is required to examine the effects of $\mathrm{p}$-Bad and the association between 14-3-3 $\zeta$ and p-Bad in LLC cells. 
Taken together, the results of the present study indicate that DC-CIK induced cell apoptosis in LLC cells, which was associated with the downregulation of $14-3-3 \xi$ and $\mathrm{p}-\mathrm{Bad}$. Whether the effects were directly associated with the modulation of autophagy, requires further study. The data presented provides evidence that DC-CIK may have the potential to serve as a promising adjuvant in the combination therapy for the treatment of NSCLC.

\section{Acknowledgements}

Not applicable.

\section{Funding}

The present study was supported by the Student's Platform for Innovation and Entrepreneurship Training Program funded by Liaoning (grant no. 201610160044).

\section{Availability of data and materials}

The datasets used and/or analyzed during the current study are available from the corresponding author on reasonable request.

\section{Authors' contributions}

DZ and XL conducted the conception and design of the present study. YH and FL performed the experiments.

\section{Ethics approval and consent to participate}

Ethical approval for the present study was obtained from the Ethics and Welfare Committee of Jinzhou Medical University (Jinzhou, China). Written informed consent was obtained from participants.

\section{Consent for publication}

Written informed consent was obtained from participants.

\section{Competing interests}

The authors declare that they have no competing interests.

\section{References}

1. Torre LA, Bray F, Siegel RL, Ferlay J, Lortet-Tieulent J and Jemal A: Global cancer statistics, 2012. CA Cancer J Clin 65: 87-108, 2015.

2. Sundar R, Soong R, Cho BC, Brahmer JR and Soo RA: Immunotherapy in the treatment of non-small cell lung cancer. Lung Cancer 85: 101-109, 2014.

3. Choudhury A, Palma M and Mellstedt $\mathrm{H}$ : The future of cancer vaccines for non-small-cell lung cancer: Ongoing trials. Clin Lung Cancer 9 (Suppl 1): S37-S44, 2008.

4. Sharma A, Moore WH, Lanuti M and Shepard JA: How I do it: Radiofrequency ablation and cryoablation of lung tumors J Thorac Imaging 26: 162-174, 2011.

5. Schiller JH, Harrington D, Belani CP, Langer C, Sandler A, Krook J, Zhu J and Johnson DH; Eastern Cooperative Oncology Group: Comparison of four chemotherapy regimens for advanced non-small-cell lung cancer. N Engl J Med 346: 92-98, 2002.

6. Koski GK, Cohen PA, Roses RE, Xu S and Czerniecki BJ: Reengineering dendritic cell-based anti-cancer vaccines Immunol Rev 222: 256-276, 2008.
7. Xie S, Wu X, Zhang G, Xu K, Bian X, Zhang S and Ye Y: Remarkable regression of a lung recurrence from an undifferentiated embryonal sarcoma of the liver treated with a DC vaccine combined with immune cells: A case report. Cell Immunol 290: 185-189, 2014.

8. Zhao P, Bu X, Wei X, Sun W, Xie X, Li C, Guo Q, Zhu D, Wei X and Gao D: Dendritic cell immunotherapy combined with cytokine-induced killer cells promotes skewing toward Th2 cytokine profile in patients with metastatic non-small cell lung cancer. Int Immunopharmacol 25: 450-456, 2015.

9. Shan CC, Shi LR, Ding MQ, Zhu YB, Li XD, Xu B, Jiang JT and Wu CP: Cytokine-induced killer cells co-cultured with dendritic cells loaded with the protein lysate produced by radiofrequency ablation induce a specific antitumor response. Oncol Lett 9: 1549-1556, 2015

10. Zhao M, Li H, Li L and Zhang Y: Effects of a gemcitabine plus platinum regimen combined with a dendritic cell-cytokine induced killer immunotherapy on recurrence and survival rate of non-small cell lung cancer patients. Exp Ther Med 7: 1403-1407, 2014.

11. Yang L, Ren B, Li H, Yu J, Cao S, Hao X and Ren X: Enhanced antitumor effects of DC-activated CIKs to chemotherapy treatment in a single cohort of advanced non-small-cell lung cancer patients. Cancer Immunol Immunother 62: 65-73, 2013.

12. Tzivion G, Gupta VS, Kaplun L and Balan V: $14-3-3$ proteins as potential oncogenes. Semin Cancer Biol 16: 203-213, 2006.

13. Neal CL and Yu D: $14-3-3 \zeta$ as a prognostic marker and therapeutic target for cancer. Expert Opin Ther Targets 14: 1343-1354, 2010.

14. Zhao J, Meyerkord CL, Du Y, Khuri FR and Fu H: 14-3-3 proteins as potential therapeutic targets. Semin Cell Dev Biol 22: 705-712, 2011.

15. Fan T, Li R, Todd NW, Qiu Q, Fang HB, Wang H, Shen J, Zhao RY, Caraway NP, Katz RL, et al: Up-regulation of 14-3-3zeta in lung cancer and its implication as prognostic and therapeutic target. Cancer Res 67: 7901-7906, 2007.

16. Lowe SW, Cepero E and Evan G: Intrinsic tumour suppression. Nature 432: 307-315, 2004.

17. Zha J, Harada H, Yang E, Jockel J and Korsmeyer SJ: Serine phosphorylation of death agonist BAD in response to survival factor results in binding to 14-3-3 not BCL-XL. Cell 87: 619-628, 1996.

18. del Peso L, González-García M, Page C, Herrera R and Nuñez G: Interleukin-3-induced phosphorylation of BAD through the protein kinase Akt. Science 278: 687-689, 1997.

19. Manegold C, Vansteenkiste J, Cardenal F, Schuette W, Woll PJ, Ulsperger E, Kerber A, Eckmayr J and von Pawel J: Randomized phase II study of three doses of the integrin inhibitor cilengitide versus docetaxel as second-line treatment for patients with advanced non-small-cell lung cancer. Invest New Drugs 31: 175-182, 2013.

20. Zhou P, Liang P, Dong B, Yu X, Han Z and Xu Y: Phase I clinical study of combination therapy with microwave ablation and cellular immunotherapy in hepatocellular carcinoma. Cancer Biol Ther 11: 450-456, 2011.

21. Lee WC, Wang HC, Hung CF, Huang PF, Lia CR and Chen MF: Vaccination of advanced hepatocellular carcinoma patients with tumor lysate-pulsed dendritic cells: A clinical trial. J Immunother 28: 496-504, 2005.

22. Zhu XP, Xu YH, Zhou J and Pan XF: A clinical study evaluating dendritic and cytokine-induced killer cells combined with concurrent radiochemotherapy for stage IIIB non-small cell lung cancer. Genet Mol Res 14: 10228-10235, 2015.

23. Cui Y, Yang X, Zhu W, Li J, Wu X and Pang Y: Immune response, clinical outcome and safety of dendritic cell vaccine in combination with cytokine-induced killer cell therapy in cancer patients. Oncol Lett 6: 537-541, 2013.

24. Li QY, Shi Y, Huang DH, Yang T, Wang JH, Yan GH, Wang HY, Tang XJ, Xiao CY, Zhang WJ, et al: Cytokine-induced killer cells combined with dendritic cells inhibited liver cancer cells. Int J Clin Exp Med 8: 5601-5610, 2015.

25. Obsil T and Obsilova V: Structural basis of 14-3-3 protein functions. Semin Cell Dev Biol 22: 663-672, 2011.

26. Gardino AK and Yaffe MB: 14-3-3 proteins as signaling integration points for cell cycle control and apoptosis. Semin Cell Dev Biol 22: 688-695, 2011.

27. Matta A, DeSouza LV, Ralhan R and Siu KW: Small interfering RNA targeting 14-3-3 increases efficacy of chemotherapeutic agents in head and neck cancer cells. Mol Cancer Ther 9: 2676-2688, 2010. 
28. Li Z,Zhao J, Du Y,Park HR, Sun SY, Bernal-Mizrachi L, Aitken A, Khuri FR and Fu H: Down-regulation of 14-3-3zeta suppresses anchorage-independent growth of lung cancer cells through anoikis activation. Proc Natl Acad Sci USA 105: 162-167, 2008.

29. Rong F, Li W, Chen K, Li DM, Duan WM, Feng YZ, Li F, Zhou XW, Fan SJ, Liu Y, et al: Knockdown of RhoGDI $\alpha$ induces apoptosis and increases lung cancer cell chemosensitivity to paclitaxel. Neoplasma 59: 541-550, 2012.

30. Lin HI, Lee YJ, Chen BF, Tsai MC, Lu JL, Chou CJ and Jow GM: Involvement of Bcl-2 family, cytochrome $\mathrm{c}$ and caspase 3 in induction of apoptosis by beauvericin in human non-small cell lung cancer cells. Cancer Lett 230: 248-259, 2005.

31. Bishopric NH, Andreka P, Slepak T and Webster KA: Molecular mechanisms of apoptosis in the cardiac myocyte. Curr Opin Pharmacol 1: 141-150, 2001.

32. Kavitha K, Kowshik J, Kishore TK, Baba AB and Nagini S: Astaxanthin inhibits NF- $\kappa B$ and $\mathrm{Wnt} / \beta$-catenin signaling pathways via inactivation of Erk/MAPK and PI3K/Akt to induce intrinsic apoptosis in a hamster model of oral cancer. Biochim Biophys Acta 1830: 4433-4444, 2013.
33. Kim MR, Jeong EG, Chae B, Lee JW, Soung YH, Nam SW, Lee JY, Yoo NJ and Lee SH: Pro-apoptotic PUMA and anti-apoptotic phospho-BAD are highly expressed in colorectal carcinomas. Dig Dis Sci 52: 2751-2756, 2007.

34. Jeong EG, Lee SH, Kim SS, Ahn CH, Yoo NJ and Lee SH: Immunohistochemical analysis of phospho-BAD protein and mutational analysis of BAD gene in gastric carcinomas. APMIS 115: 976-981, 2007.

35. Zippo A, Serafini R, Rocchigiani M, Pennacchini S, Krepelova A and Oliviero S: Histone crosstalk between H3S10ph and H4K16ac generates a histone code that mediates transcription elongation. Cell 138: 1122-1136, 2009. 